\title{
Fetal DHA inadequacy and the impact on child neurodevelopment: a follow-up of a randomised trial of maternal DHA supplementation in pregnancy
}

\author{
Kelly A. Mulder ${ }^{1}$, Rajavel Elango ${ }^{1,2 *}$ and Sheila M. Innis ${ }^{1 *}$ \\ ${ }^{1}$ Department of Paediatrics, BC Children's Hospital Research Institute, Faculty of Medicine, University of British Columbia, \\ Vancouver, BC, Canada V5Z 4 H4 \\ ${ }^{2}$ School of Population and Public Health, University of British Columbia, Vancouver, BC, Canada V6T 1Z3
}

(Submitted 16 July 2017 - Final revision received 21 October 2017 - Accepted 17 November 2017-First published online 10 January 2018)

\section{Abstract}

DHA is an important component of neural lipids accumulating in neural tissue during development. Inadequate DHA in gestation may compromise infant development, but it is unknown whether there are lasting effects. We sought to determine whether the observed effects of fetal DHA inadequacy on infant development persist into early childhood. This follow-up study assessed children (5-6 years) whose mothers received $400 \mathrm{mg} / \mathrm{d}$ DHA or a placebo during pregnancy. Child neurodevelopment was assessed with several age-appropriate tests including the Kaufman Assessment Battery for Children. A risk-reduction model was used whereby the odds that a child from the maternal placebo group would fail to achieve a test score in the top quartile was calculated. The association of maternal DHA intake and status in gestation with child test scores, as well as with child DHA intake and status, was also determined. No differences were detected in children ( $n$ 98) from the maternal placebo and DHA groups achieving a high neurodevelopment test score $(P>0.05)$. However, maternal DHA status was positively related to child performance on some tests including language and short-term memory. Furthermore, child DHA intake and status were related to the mother's intake and status in gestation. The neurodevelopment effects of fetal DHA inadequacy may have been lost or masked by other variables in the children. Although we provide evidence that maternal DHA status is related to child cognitive performance, the association of maternal and child DHA intake and status limits the interpretation of whether DHA before or after birth is important.

\section{Key words: Fetal DHA inadequacy: Child brain development: Maternal DHA intake: Child DHA intake}

The $n$-3 fatty acid DHA (22:6n-3, DHA) increases in the brain grey matter and retina phospholipids (PL) during early development, with a well-known higher proportion of DHA in the brain and retina PL than other organs. Loss of brain DHA results in replacement with the $n-6$ fatty acids $22: 4 n-6$ and $22: 5 n-6$ in both animals and humans ${ }^{(1,2)}$, which has been associated with impaired or reduced visual acuity and aspects of neurological function in animals ${ }^{(1-4)}$. Several randomised intervention trials have reported higher visual acuity and neurological outcomes for infants and children born to mothers given DHA during gestation compared with a placebo ${ }^{(5-7)}$, whereas others have found no benefit ${ }^{(8-10)}$ or a negative effect ${ }^{(11,12)}$. Similarly, several studies have provided evidence that addition of DHA to infant formula fed in the weeks or months after birth leads to greater visual and neurological development in term and preterm infants compared with formula with no $\mathrm{DHA}^{(13-18)}$.

It is well known that nutritional deficiency of certain essential nutrients during development can have lasting, irreversible effects on the brain, which do not occur when the same deficiency occurs later on. For example, folate deficiency occurring within $28 \mathrm{~d}$ post-conception may interfere with closure of the neural tube ${ }^{(19)}$, whereas iodine deficiency before birth is associated with irreversible neurological consequences ${ }^{(20)}$. Deficiency of DHA in the brain may be caused by an inadequate source of DHA or its precursor $\alpha$-linolenic acid (18:3n-3), which synthesises DHA via EPA (20:5n-3, EPA) through desaturation and elongation ${ }^{(21)}$. A high intake of the $n-6$ fatty acid linoleic acid $(18: 2 n-6)$ has also been shown to be associated with DHA deficiency, with increased arachidonic acid $(20: 4 n-6)$ in the brain during early development ${ }^{(22)}$. Loss of neural DHA when an $n-3$ fatty acid-deficient or high $n-6$ fatty acid diet is fed to the mother during gestation has also been shown to alter neurotransmitter metabolism, neural function and behaviour in animals, including rodents and non-human primates ${ }^{(1,3,23,24)}$.

The infant brain, however, is only about $25 \%$ of the adult brain weight at term gestation, being about $370 \mathrm{~g}$ and increasing to about $70 \%$ of adult brain weight by 12 months ${ }^{(25)}$. Synapses, which are rich in DHA, increase with remarkable rates of

Abbreviations: Beery, Beery-Buktenica Developmental Test of Visual-Motor Integration; IQ, intelligence quotient; MPI, Mental Performance Index; PC, phosphatidylcholine; PE, phosphatidylethanolamine; PPVT, Peabody Picture Vocabulary Test.

* Corresponding author: R. Elango, email relango@bcchr.ubc.ca

\& Deceased. 


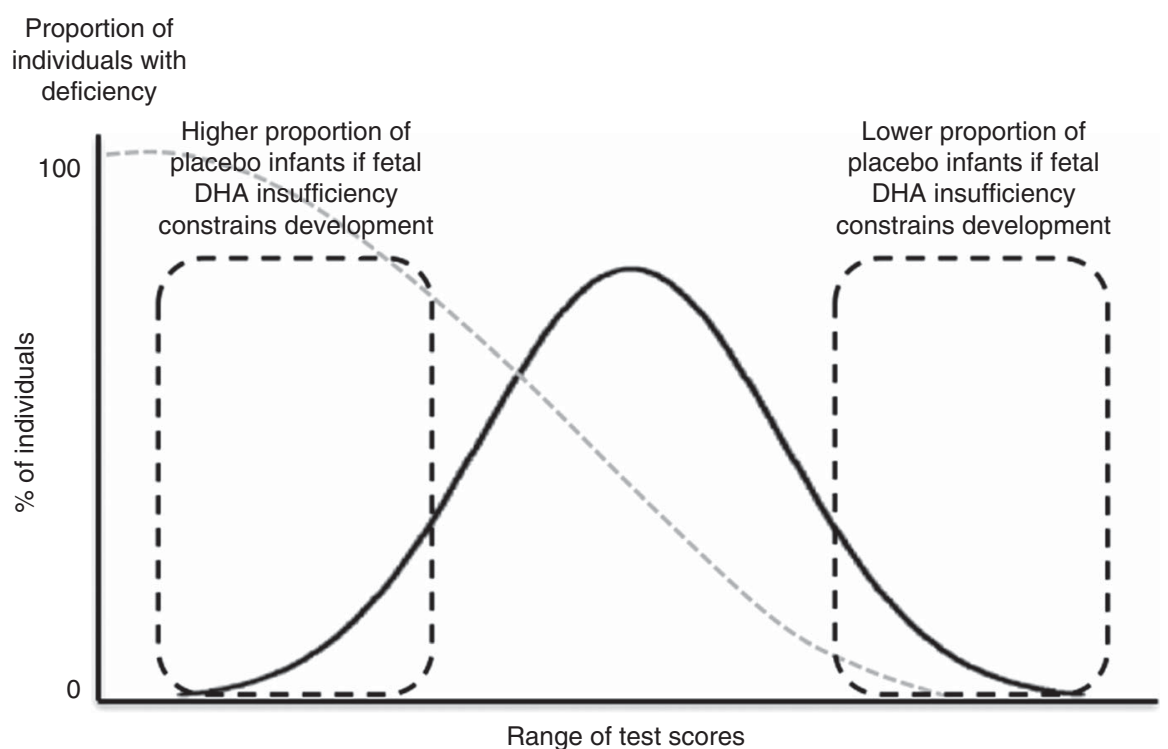

Fig. 1. Illustration to highlight our assumption that children with high neurodevelopment test scores were unlikely to be nutrient deficient in utero. The potential longterm effects of fetal DHA insufficiency were assessed as the risk that a child from the maternal placebo group would fail to achieve a test score in the highest quartile of neurodevelopment test scores.

formation of 40000 synapses/second during the first 2 years, with structural reorganisation and pruning of synapses leading to maximum synaptic density from about 6-7 years ${ }^{(26)}$. Both observational and randomised intervention studies have provided evidence that DHA intake and status of children up to about 10 years are also related to child neural function, including verbal learning ability ${ }^{(27)}$, language ${ }^{(28)}$, reading ${ }^{(29,30)}$, spelling ${ }^{(29)}$, non-verbal intelligence ${ }^{(29,31)}$ and memory ${ }^{(32)}$. Reaction time and prefrontal cortex activation during a sustained attention test has also been associated with baseline erythrocyte DHA in boys aged 8-10 years, and 400 or $1200 \mathrm{mg} / \mathrm{d}$ DHA for 8 weeks led to increased prefrontal cortex activation from baseline compared with controls ${ }^{(33)}$. This suggests that although DHA deficiency during gestation may have lasting effects, continued brain development after birth with child diet potentially impacting brain PL DHA may in turn impact child brain function.

We previously reported that DHA insufficiency in some pregnant women in our population was low enough to limit infant neurodevelopment to 18 months of age ${ }^{(34)}$. The primary purpose of the current study was to determine whether fetal DHA insufficiency extends to neurological effects in children assessed at 5.75 years. The analysis of both the previous and the present studies are based on the concept that infants with the highest scores on neurodevelopmental tests were unlikely to be nutrient deficient (Fig. 1). In addition, this study also sought to determine whether the child's DHA intake and erythrocyte status was related to the mother's DHA intake and erythrocyte status during gestation.

\section{Methods}

\section{Subjects and study design}

This was a follow-up of children born to mothers who participated in a randomised, double-blind, placebo-controlled study to assess whether DHA deficiency during pregnancy occurs and is sufficient to limit infant central nervous system (CNS) development up to 18 months of age ${ }^{(34)}$. In brief, pregnant women were randomised to $400 \mathrm{mg} / \mathrm{d}$ DHA or a placebo from 16 weeks of gestation until delivery, with follow-up only of singleton, termgestation infants, with no congenital or metabolic diseases likely to affect infant neurological development. A total of 200 infants, ninety-six and 104 for maternal DHA or placebo supplementation groups, respectively, were invited for follow-up. Of these, ninetyeight parents agreed to participate in this follow-up study that assessed dietary intake, DHA status and CNS development in children at 5.75 years. New computer-generated codes were made for all participants to avoid knowledge of the gestational intervention group and infant outcome data to 18 months.

The present study was conducted according to guidelines laid down in the Declaration of Helsinki, with written informed consent obtained from a parent or guardian for each child before any assessment or data collection. All procedures involving human subjects in this study were approved by the Committee for Ethical Review of Research Involving Human Subjects at the University of British Columbia and the British Columbia Children's and Women's Hospital.

\section{Subject characteristics, dietary assessment and biochemical analysis}

Family and socio-demographic information, including income, education and number of adults and children in the home, was updated by questionnaire. Parental intelligence quotient (IQ) was assessed with the Test of Nonverbal Intelligence-3rd edition, which assesses aptitude, abstract reasoning and problem-solving, without dependence on language or formal education ${ }^{(35)}$. Each child weight and height was measured, and then weight, height and BMI $z$ scores calculated using the WHO Anthroplus anthropometric calculator (version 1.0.4). 
Dietary intake was assessed using an FFQ by face-to-face interview with the parent, using food models and measuring utensils, including all foods and beverages consumed by the child over the previous 4 weeks. Dietary intake of macronutrients and fatty acids were quantified using nutrient analysis software (ESHA Food Processor SQL, version 10.10.0.0; ESHA Research), ensuring that the database had complete and accurate fatty acid compositions based on product labels for our location, and where necessary our laboratory analysed the food fatty acids.

Child venous blood $(6 \mathrm{ml})$ was collected with EDTA, centrifuged $\left(2500 \mathrm{~g}, 10 \mathrm{~min}, 4^{\circ} \mathrm{C}\right)$, the plasma and buffy coat separated, aliquoted and frozen at $-80^{\circ} \mathrm{C}$ within $30 \mathrm{~min}$ of blood collection. The erythrocytes were washed by re-suspension with saline and EDTA, centrifuged twice and then the erythrocytes were frozen at $-80^{\circ} \mathrm{C}$ until analysis. Erythrocyte total lipids were extracted, then fatty acids quantified by routine $\mathrm{GLC}^{(36)}$. Preparation of blood and analysis of erythrocytes was led by technician D. J. King, an expert with clinical samples for fatty acids.

\section{Child neural development}

Child neural development was assessed using age-appropriate standardised tests, in a dedicated room free of distractions, with a one-way mirror to enable the parent to view their child. The tests included the Kaufman Assessment Battery for Children, 2nd edition (KABC), which enables scaled subset scores to assess cognitive ability. The sequential processing scale measures short-term memory; the learning ability scale measures long-term storage and retrieval; and the simultaneous processing scale measures visual processing. General mental processing ability is also measured by combining all three scales to give a composite Mental Performance Index (MPI) score ${ }^{(37)}$. The KABC delayed recall was also done, which measures long-term memory. Language development using the Peabody Picture Vocabulary Test- 4 (PPVT) ${ }^{(38)}$ and visual-motor integration using the Beery-Buktenica Developmental Test of Visual-Motor Integration (Beery) $)^{(39)}$ were also assessed. Finally, the Test of Variables of Attention (TOVA) ${ }^{(40)}$ was used to assess attention and impulsivity, measured by the scores of errors of commission and omission, respectively, and also response time, a measure of processing speed, and response time variability, a measure of response time consistency.

\section{Data analysis}

We used descriptive statistics to summarise subject characteristics, and compared variables between the DHA supplement and placebo groups using independent $t$ tests, Mann-Whitney $U$ tests or $\chi^{2}$ tests, as appropriate. Family and child characteristics including infant birth weight, sex and human milk-feeding duration, maternal IQ, number of children and adults in the home were screened for potential associations with child neurodevelopment test scores.

The potential impact of gestation was assessed using contingency tables and Fisher exact tests to determine the OR and $95 \%$ CI that a child from the placebo group would fail to score in the top quartile of children for each test. Where children could not be separated into an exact upper quartile owing to several children with the same score, a cut-off point 'quartile' giving the lowest deviation from $25 \%$ of the group was used. Our previous report showed a considerable overlap in maternal DHA status, measured as erythrocyte phosphatidylethanolamine (PE) and phosphatidylcholine (PC) DHA, between the DHA and placebo groups after approximately 20 weeks of supplementation during pregnancy ${ }^{(34)}$. Thus, Pearson's correlation or Spearman's rank correlation coefficient, as appropriate, were used to determine the potential relationship between maternal DHA status at 16 and 36 weeks of gestation and child performance on cognitive tests.

The potential association between the mother's prenatal dietary DHA intake and DHA status with the children's DHA intake and DHA status at $\mathbf{5 . 7 5}$ years was assessed using Pearson's correlation or Spearman's rank correlation coefficient, as appropriate. Owing to the $400 \mathrm{mg} / \mathrm{d}$ DHA supplementation from 16 weeks of gestation to term infant birth, the relationship between the maternal erythrocyte DHA, 22:4n-6, 22:5n-6 and the DHA:22: $4 n-6+22: 5 n-6$ ratio was addressed for all children at 16 weeks of gestation, but not at 36 weeks of gestation. Data analysis was done using IBM SPSS Statistics (version 20.0.0, 2011), with significance set at $P<0.05$.

\section{Results}

A total of ninety-eight children, aged 5.75 years, from the study on DHA deficiency during gestation ${ }^{(34)}$ participated in this follow-up study from 2010 to 2014. No significant differences were found in maternal or infant characteristics at birth or through the 18-month infant follow-up between children who did not participate in this study ( $n$ 102) and those who did ( $n$ 98). The child and family characteristics are shown in Table 1 . For the group, $74.5 \%$ of the children were Caucasian, $12.2 \%$ were Chinese and $13.3 \%$ were of other ethnicities; of the children, $53.1 \%$ were girls, $83.5 \%$ of the children lived with two adults, $55 \cdot 1 \%$ had one sibling, $89.8 \%$ were human milk-fed at least 3 months and had a BMI $z$ score mean 0.06 (SD 1.01) at 5.75 years. No differences were found in maternal IQ measures, family characteristics or the child sex, length of human milk-feeding, weight, height or BMI assessment, $P>0 \cdot 05$, between children in the placebo ( $n$ 52) and DHA ( $n$ 46) groups. Cognitive tests were completed for $>90 \%$ of children, with incomplete tests for PPVT, $n$ 1; Kaufman ABC sequential, $n$ 2; learning, $n$ 4; simultaneous, $n 1$; MPI, $n$ 5; delayed recall, $n 1$; Beery, $n 0$; TOVA, $n$ 9. The FFQ was analysed for all children, and blood samples sufficient for fatty acid analysis were obtained from seventy-three children.

Potential variables associated with child neural development were screened for all cognitive tests and showed a lower reaction time $(P=0.003)$ and higher percent errors of commission $(P=0.004)$ in boys than girls on the TOVA, and a higher PPVT scores for Caucasian than non-Caucasian children $(P=0 \cdot 001)$. Infant human milk-feeding duration showed no association with child development test results; however, only $10 \cdot 2 \%$ of the children were human milk-fed $<3$ months. There was no effect of any other collected or measured child or family characteristic on child cognitive scores at 5.75 years.

No significant differences were found in mean test performance between children in the maternal gestation DHA and 
Table 1. Family and child characteristics (Mean values and standard deviations; percentages)

\begin{tabular}{|c|c|c|c|c|}
\hline & All $(n 98)$ & Placebo ( $n$ 52) & DHA $(n 46)$ & $P^{*}$ \\
\hline Ethnicity, Caucasian/non-Caucasian (\%) & $74.5 / 25.5$ & $76 \cdot 4 / 23 \cdot 6$ & $71 \cdot 7 / 28 \cdot 3$ & 0.826 \\
\hline Maternal TONI IQ & & & & 0.202 \\
\hline Mean & $36 \cdot 0$ & $37 \cdot 0$ & 34.9 & \\
\hline SD & 6.90 & $6 \cdot 85$ & $6 \cdot 89$ & \\
\hline Adults in home, $>2 / 2 / 1(\%)$ & $11 \cdot 3 / 83 \cdot 5 / 5 \cdot 15$ & $13 \cdot 7 / 80 \cdot 4 / 5 \cdot 88$ & $8 \cdot 70 / 87 \cdot 0 / 4 \cdot 35$ & 0.679 \\
\hline Children in home, $>2 / 2 / 1(\%)$ & $30 \cdot 9 / 55 \cdot 7 / 13 \cdot 4$ & $9 \cdot 80 / 66 \cdot 7 / 23 \cdot 5$ & $17 \cdot 4 / 43 \cdot 5 / 39 \cdot 1$ & 0.071 \\
\hline Child sex, boys/girls (\%) & $46 \cdot 9 / 53 \cdot 1$ & $53 \cdot 8 / 46 \cdot 2$ & $39 \cdot 1 / 60 \cdot 9$ & 0.161 \\
\hline Breast-fed $>3$ months $(\%)$ & $89 \cdot 8$ & $92 \cdot 3$ & $87 \cdot 0$ & 0.508 \\
\hline BMI-for-age, $z$ score & & & & 0.730 \\
\hline Mean & 0.06 & $0 \cdot 10$ & 0.03 & \\
\hline SD & 1.01 & 1.02 & 1.00 & \\
\hline
\end{tabular}

TONI, Test of Nonverbal Intelligence.

* Statistical comparison between the prenatal maternal placebo and DHA groups.

Table 2. Cognitive scores for all children and by maternal DHA supplement or placebo group* (Mean values and standard deviations; medians and 2.5-97.5 percentiles)

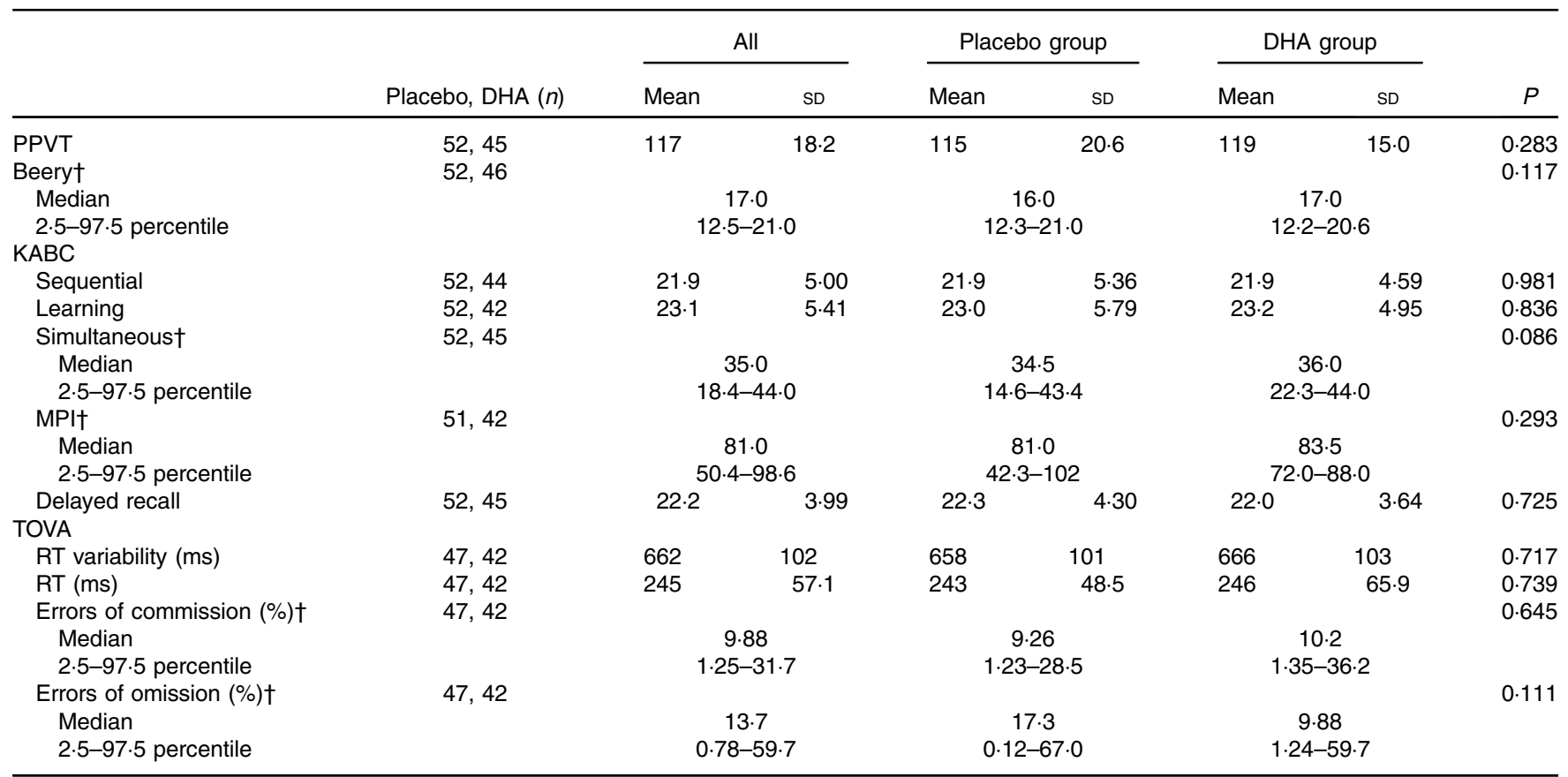

PPVT, Peabody Picture Vocabulary Test; Beery VMI, Beery-Buktenica Developmental Test of Visual-Motor Integration; KABC, Kaufman Assessment Battery for Children, 2nd edition; MPI, Mental Performance Index; TOVA, Test of Variables of Attention; RT, response time.

* Compared by Student's $t$ test or Mann-Whitney $U$ test.

† Data are not normally distributed.

placebo groups (Table 2). We also found no differences between children in the two maternal groups in achieving performance in the upper quartile of test performance (Table 3); PPVT $(P=1 \cdot 00)$, Beery $(P=1 \cdot 00)$, the KABC sequential processing $(P=0 \cdot 808)$, learning ability $(P=0.628)$, simultaneous processing $(P=0.818)$, MPI $(P=0.476)$ and delayed recall $(P=0.825)$, or on the TOVA response time variability $(P=0.739)$, response time $(P=0.717)$, errors of commission $(P=0.645)$ and errors of omission $(P=0 \cdot 111)$.

There were no associations between the maternal prenatal dietary DHA and child cognitive test scores (online Supplementary Table S1). The assessment of maternal erythrocyte DHA status during gestation and child cognitive performance are shown in the online Supplementary Tables S2 and S3. The children's Beery score was positively associated with maternal DHA/22: $4 n-6+22: 5 n-6$ in erythrocyte PE at 16 weeks of gestation $(\rho=0.247, P=0.016)$ and 36 weeks of gestation $(\rho=0.221$, $P=0.032)$, and inversely associated with maternal 36-week erythrocyte PE $22: 4 n-6(\rho=-0 \cdot 247, P=0 \cdot 016)$ (online Supplementary Table S2). The children's Beery scores were also associated with maternal erythrocyte PC fatty acids of interest at 16 weeks of gestation and are shown in the online Supplementary Table S2.

As previous studies have shown associations between the maternal and child diet ${ }^{(41-44)}$, we addressed the relationship of child DHA intake and erythrocyte DHA to that of the mother's. DHA intake among the children was skewed, with a median 
Table 3. Risk that a child in the placebo group would be in the lowest quartile rather than the highest quartile of cognitive development test scores

(Odds ratios and $95 \%$ confidence intervals)

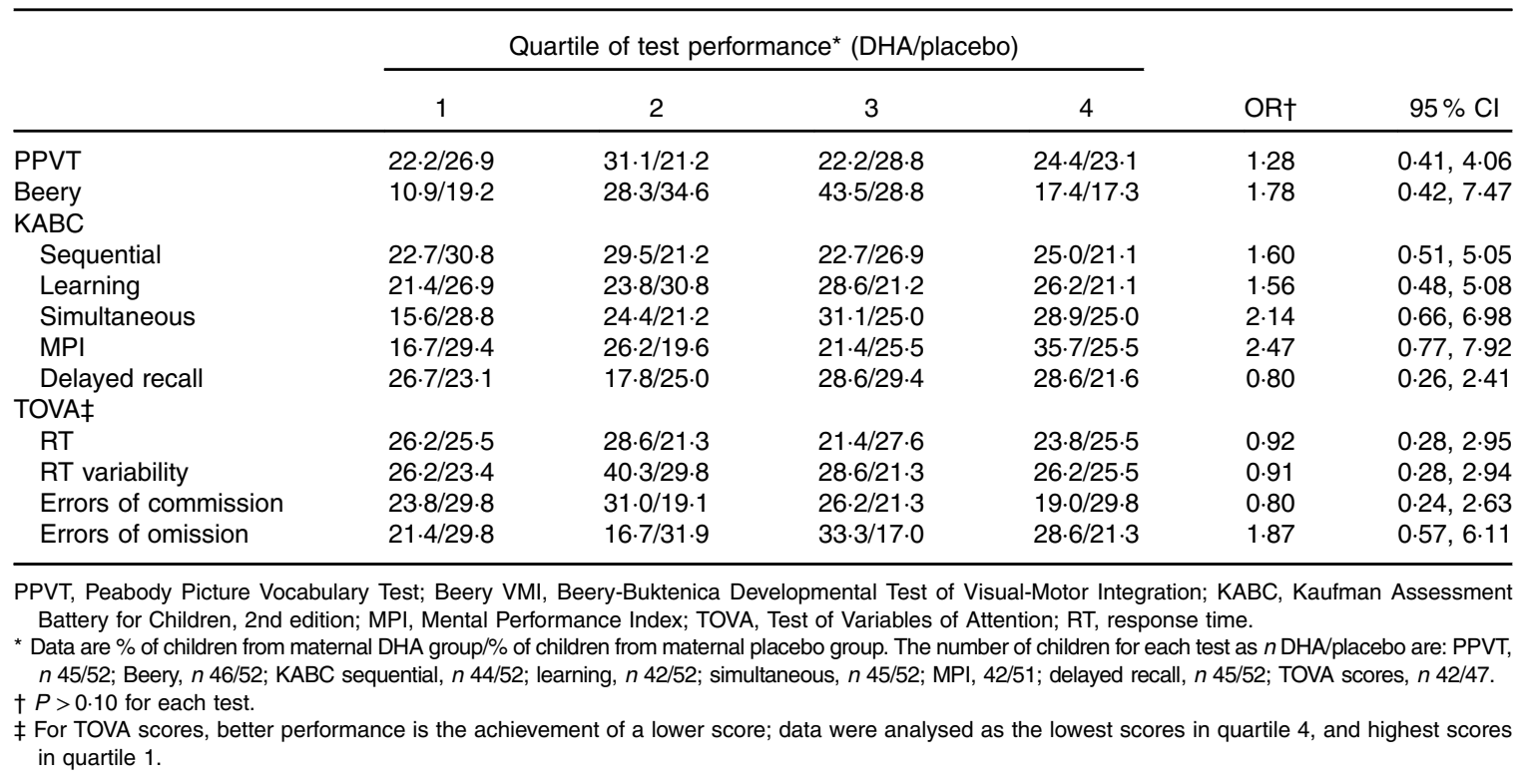

$(2 \cdot 5-97.5 \%)$ of $43.2 \mathrm{mg} / \mathrm{d}(0-300 \mathrm{mg} / \mathrm{d}$ ) (online Supplementary Table S4). There was no difference in DHA intake between boys and girls $(P=0.524)$, or between children in the prenatal placebo and DHA groups $(P=0.603)$. The child DHA intake at 5.75 years was significantly related to their mother's DHA intake at both 16 weeks and 36 weeks of gestation, $\rho=0.317, P=0.002$ and $\rho=0.256, P=0 \cdot 013$, respectively (Table 4 ). In addition, the children's DHA intake was related to maternal erythrocyte PE and PC DHA/22: $4 n-6+22: 5 n-6$ at 16 weeks of gestation, $\rho=0 \cdot 297$, $P=0.004$ and $\rho=0.264, P=0.010$, respectively, $(n$ 94) (Table 4).

Child erythrocyte DHA mean was 5.20 (sD 1.43) \%TFA, with 3.31 (SD 0.68) and 0.63 (SD 0.16) $22: 4 n-6$ and $22: 5 n-6$, respectively, and erythrocyte DHA/22:4n-6+22:5n-6 of median (2.5-97.5\%) $1.31(0 \cdot 60-3 \cdot 34)$ \%TFA. Child erythrocyte DHA was significantly related to child DHA intake $(\rho=0.396, P<0.001)$. The relationship between child erythrocyte DHA at 5.75 years and maternal DHA intake and erythrocyte DHA at 16 weeks of gestation is shown in Table 5. Child erythrocyte DHA was related to maternal DHA intake at 36 weeks of gestation $(\rho=0.277$, $P=0 \cdot 021)$, but not at 16 weeks of gestation $(\rho=0 \cdot 195, P=0 \cdot 109)$. The child erythrocyte DHA was related to maternal 16-week erythrocyte PE DHA $(\rho=0 \cdot 239, P=0 \cdot 048)$, erythrocyte PE $22: 5 n-6(\rho=-0.286, P=0.017)$ and erythrocyte PE DHA/ $22: 4 n-6+22: 5 n-6(\rho=0 \cdot 313, P=0 \cdot 009)$, but not erythrocyte PE $22: 4 n-6(r 0 \cdot 124, P=0 \cdot 312)$. Child erythrocyte DHA was also related to erythrocyte PC DHA/22:4n-6+22:5n-6 $(\rho=0 \cdot 306$, $P=0.010)$, but not erythrocyte PC DHA $(r 0.219, P=0.375)$, erythrocyte PC $22: 4 n-6(\rho=-0 \cdot 080, P=0.512)$ or erythrocyte PC $22: 5 n-6(\rho=-0 \cdot 120, P=0 \cdot 323)$ at 16 weeks of gestation.

\section{Discussion}

We used a risk-reduction model to address the potential longterm effect of DHA during gestation on cognitive performance
Table 4. Associations of child dietary DHA with maternal dietary and erythrocyte markers of DHA sufficiency during gestation

\begin{tabular}{lcc}
\hline & \multicolumn{2}{c}{ Child DHA intake* $^{*}$} \\
\cline { 2 - 3 } Mothers & $\rho$ & $P$ \\
\hline 16-week gestation DHA intake & 0.317 & 0.002 \\
36-week gestation DHA intake & 0.256 & 0.013 \\
16-week gestation erythrocyte PC & & \\
DHA & 0.074 & 0.481 \\
22:4n-6 & -0.191 & 0.065 \\
22:5n-6 & -0.183 & 0.077 \\
DHA/22:4n-6+22:5n-6 & 0.264 & 0.010 \\
16-week gestation erythrocyte PE & & \\
DHA & 0.197 & 0.057 \\
$22: 4 n-6$ & -0.138 & 0.183 \\
22:5n-6 & -0.199 & 0.054 \\
DHA/22:4n-6+22:5n-6 & 0.297 & 0.004 \\
\hline
\end{tabular}

PC, phosphatidylcholine; PE, phosphatidylethanolamine.

* Data are Spearman's rank correlation coefficients, for all mother-child pairs with available dietary DHA and erythrocyte fatty acids, $n 69$.

when assessed at 5.75 years (Fig. 1). Although we found that DHA insufficiency during gestation was associated with a greater risk of failure to achieve a high score in infant neurodevelopment tests to 18 months ${ }^{(34)}$, evidence of lasting effects in children up to 5.75 years was not detected. The apparent effects of DHA insufficiency during gestation on CNS development up to 18 months of age may have been lost or masked by postnatal dietary or other variables in children at 5.75 years. The potential importance of the child's postnatal diet to continued brain development and the association between the children's dietary habits and that of the mother raises the question of the implications of epidemiological pregnancy studies that consider only the maternal diet or status on long-term child outcome. 
Table 5. Associations of maternal dietary and erythrocyte DHA during gestation with the children's erythrocyte markers of DHA sufficiency

\begin{tabular}{|c|c|c|c|c|c|}
\hline \multirow[b]{2}{*}{ Mothers } & & \multicolumn{4}{|c|}{ Child erythrocyte fatty acids* } \\
\hline & & $\mathrm{DHA}$ & $22: 4 n-6$ & $22: 5 n-6$ & $\mathrm{DHA} / 22: 4 n-6+22: 5 n-6$ \\
\hline \multicolumn{6}{|l|}{ Maternal DHA intake } \\
\hline \multirow[t]{2}{*}{ 16-week gestation } & $\rho$ & 0.195 & -0.292 & -0.333 & 0.301 \\
\hline & $P$ & 0.109 & 0.015 & 0.005 & 0.012 \\
\hline \multirow[t]{2}{*}{ 36-week gestation } & $\rho$ & 0.277 & -0.342 & -0.233 & 0.381 \\
\hline & $P$ & 0.021 & 0.004 & 0.055 & 0.001 \\
\hline \multicolumn{6}{|c|}{ 16-week gestation erythrocyte PC } \\
\hline \multirow[t]{2}{*}{$\mathrm{DHA}$} & $\rho$ & 0.219 & -0.197 & -0.208 & 0.393 \\
\hline & $P$ & 0.375 & 0.101 & 0.084 & 0.001 \\
\hline \multirow[t]{2}{*}{$22: 4 n-6$} & $\rho$ & -0.080 & 0.091 & 0.104 & -0.099 \\
\hline & $P$ & 0.512 & 0.453 & 0.390 & 0.414 \\
\hline \multirow[t]{2}{*}{$22: 5 n-6$} & $\rho$ & -0.120 & 0.141 & 0.153 & -0.148 \\
\hline & $P$ & 0.323 & 0.244 & 0.206 & 0.220 \\
\hline \multirow[t]{2}{*}{$\mathrm{DHA} / 22: 4 n-6+22: 5 n-6$} & $\rho$ & 0.306 & -0.206 & -0.194 & 0.333 \\
\hline & $P$ & 0.010 & 0.087 & $0 \cdot 107$ & 0.005 \\
\hline \multicolumn{6}{|c|}{ 16-week gestation erythrocyte PE } \\
\hline \multirow[t]{2}{*}{$\mathrm{DHA}$} & $\rho$ & 0.239 & -0.065 & 0.077 & 0.226 \\
\hline & $P$ & 0.048 & 0.594 & 0.531 & 0.062 \\
\hline \multirow[t]{2}{*}{$22: 4 n-6$} & $\rho$ & -0.124 & 0.373 & 0.221 & -0.329 \\
\hline & $P$ & 0.312 & 0.002 & 0.068 & 0.006 \\
\hline \multirow[t]{2}{*}{$22: 5 n-6$} & $\rho$ & -0.286 & 0.279 & 0.278 & -0.392 \\
\hline & $P$ & 0.017 & 0.020 & 0.021 & 0.001 \\
\hline \multirow[t]{2}{*}{$\mathrm{DHA} / 22: 4 n-6+22: 5 n-6$} & $\rho$ & 0.313 & 0.312 & -0.117 & 0.400 \\
\hline & $P$ & 0.009 & 0.009 & 0.337 & 0.001 \\
\hline
\end{tabular}

PC, phosphatidylcholine; PE, phosphatidylethanolamine.

* Data are Spearman's rank correlation coefficients, for all mother-child pairs with available dietary DHA and erythrocyte fatty acids, $n 69$.

The primary purpose of the present study was to determine whether DHA insufficiency during gestation had long-term effects on neurological development when assessed in a prospective group of children at 5.75 years. In our previous report, we found that infants from the placebo group were at an increased risk of not achieving a high visual acuity score at 2 months, although this was not detected at 12 months ${ }^{(34)}$. Infants from the placebo group were also at increased risk of not achieving a score in the highest quartile for tests of language development at 14 and 18 months $^{(34)}$. At 5.75 years, there was no difference in risk of failure to achieve a high score between maternal DHA and placebo groups on the language test (PPVT) $(P=1.00)$, or any other cognitive test assessed $(P>0.05)$. Although studies of the effect of DHA supplementation during pregnancy on infant outcome have reported mixed results, the majority of studies on long-term outcome from 2 to 7 years have reported no benefit of maternal DHA supplementation during pregnancy on tests of child attention ${ }^{(45)}$, working memory and inhibitory control ${ }^{(45)}$, general mental processing ability (KABC MPI), short-term memory (KABC Sequential) and visual processing (KABC Simultaneous) ${ }^{(46)}$, general cognitive function ${ }^{(11)}$ or the Hempel and Touwen neurological examinations ${ }^{(47)}$. In contrast, one study found higher mean scores for hand-eye coordination in children aged 2.5 years $(n 72)$ born to mothers taking fish oil during pregnancy compared with a placebo ${ }^{(7)}$. One other study reported a slightly higher mean score on general mental processing ability (KABC MPI) in a subset of children at 4 years ( $n 84)$ whose mothers had taken cod liver oil compared with a placebo during pregnancy $(P=0.049)^{(6)}$, although this was not seen when the children were assessed at 7 years $(n 143)^{(46)}$.
In most DHA interventions, the placebo groups do not have 'non-exposure,' which leads to overlap of nutrient intake and status with the DHA supplementation group. Although a higher amount of supplemental DHA may mitigate the overlap to some extent, nutrient intake exceeding individual requirements should not provide additional benefit. Thus, our study differs from efficacy studies in that it was designed to detect whether fetal DHA deficiency extended to 5.75 years, and not to show that DHA supplements during gestation enhance neurodevelopment assessed in childhood. As both the DHA and placebo groups in our study would likely contain individuals who were not deficient in gestation and hence unable to respond to intervention, we did not expect to find differences in mean scores between the groups. It is possible that an intervention in a population with a high prevalence of DHA deficiency may enable the detection of an efficacious response to increased DHA intake. However, a biomarker of DHA insufficiency is currently unavailable.

In the present study, visual-motor integration (Beery) scores were negatively associated with maternal prenatal erythrocytes $22: 4 n-6$ and $22: 5 n-6$. Both $22: 4 n-6$ and $22: 5 n-6$ have been shown to accumulate in the brain when brain DHA is low ${ }^{(2,48)}$. In animals, increased brain $22: 4 n-6$ and $22: 5 n-6$ is associated with functional consequences ${ }^{(1)}$, but it is unknown whether the negative effects are attributable to low brain DHA or the high long-chain $n-6$ fatty acids. A SNP in the fatty acid desaturase (FADS) and elongase (ELOVL) genes may also limit endogenous synthesis of DHA; however, 20:4n-6, 22:4n-6 and 22: $5 n-6$ may depend on the same enzymes for synthesis ${ }^{(49,50)}$. Whether or not SNPs in FADS and ELOVL genes may protect the brain from accumulating $22: 4 n-6$ and $22: 5 n-6$ in the absence 
of DHA is unclear, and thus further work is required to determine whether the effects of low brain DHA are observed and whether levels of $22: 4 n-6$ and $22: 5 n-6$ are proportionately low.

The brain of a healthy term-born infant doubles its birth weight (about $370 \mathrm{~g}$ ) in the first six postnatal months ${ }^{(25)}$, and DHA to support continuing brain growth must be provided by $n-3$ fatty acids in human milk or infant formula. Accretion of DHA in the infant brain continues rapidly after birth for about 2 years, with brain PL DHA increasing until approximately 8 years $^{(22,51)}$. During this period, the brain undergoes morphological and functional changes including synaptic proliferation, remodelling and pruning ${ }^{(52)}$, but whether prenatal DHA insufficiency alone is sufficient to alter these processes is unknown. The DHA intake of the present group of children was highly skewed, with a median intake of $43.2 \mathrm{mg} / \mathrm{d}$ and $2.5-97.5 \%$ intake of $0-300 \mathrm{mg} / \mathrm{d}$. The DHA intake of children in this study is within the range of international estimates for this age group, but the interquartile range (IQR) of $17 \cdot 4-97 \cdot 4 \mathrm{mg} / \mathrm{d}$ is higher than the population IQR for DHA intake of US children aged $1-5$ years of $10 \cdot 8-26 \cdot 2 \mathrm{mg} / \mathrm{d}(n 2496)^{(53)}$. Although it is not known whether the level of intake is sufficient for continued brain development of the young child, positive associations have been found between neurodevelopment and both $n$-3 intake and DHA status in children up to 10 years $^{(28,31,32,54)}$. A recent study showed positive associations between plasma DHA at age 7 years and assessments of reading $(\beta=0 \cdot 158$, $P<0 \cdot 001)$ and spelling $(\beta=0 \cdot 146, P=0 \cdot 001)^{(54)}$. The children's reading and spelling scores were not significantly related to cord blood plasma DHA or maternal plasma DHA in gestation.

The present study highlights the challenge in understanding whether long-term effects on outcomes, particularly those with a developmental time course extending beyond infancy, are explained by dietary intake during pregnancy or by the child's own diet, if children's diets reflect the diet of the mother. The associations between maternal prenatal and child DHA intake and status suggest dietary and potentially genetic similarities between mothers and children. Maternal DHA intake at 16 weeks and 36 weeks of gestation was positively associated with child DHA intake, and maternal DHA intake at 36 weeks of gestation was positively associated with child erythrocyte DHA at 5.75 years. Although the association between the maternal and child DHA intakes have not previously been published, several studies have reported a relationship between the maternal and child diet ${ }^{(41-43)}$, with associations stronger for the maternal-child diet than the paternal-child diet ${ }^{(41,42)}$. The Framingham Children's study reported that at 3-5 years the child's polyunsaturated fat intake was positively associated with the mother's ( $r 0.33, P \leq 0.01, n$ 87) but not the father's intake ( $r 0 \cdot 10, P>0 \cdot 05, n$ 83), with similar findings for several other nutrients $^{(42)}$. Similarly, the ALSPAC study reported a positive association between the child's intake at 10 years and the maternal prenatal ( $n$ 5717) and postnatal ( $n$ 5593) diet for protein, carbohydrate and fat, but only child protein intake was associated with the paternal diet $\left(n\right.$ 3009) ${ }^{(41)}$. Notably, the relationship between child and maternal prenatal total fat intake was stronger than the postnatal intake ${ }^{(41)}$. For a subsample of a prospective cohort study in Italy, fish and seafood intake was lower in the prenatal diet than the postnatal diet, but there was no difference in fish intake between mother's postnatal diet and fish intake of the children at 8-11 years $(n 37)^{(43)}$.

In conclusion, the results of the present study have shown that the effects of maternal DHA during gestation observed in infants to 18 months were not identified at 5.75 years. The potential long-term effects of DHA insufficiency may be too small to detect with our experimental design, or it is possible that the DHA intake of our population was not low enough during gestation to have lasting effects. Human brain development begins as early as the third week of gestation, and while considerable DHA accretion has been reported to occur during the brain growth spurt beginning in the third trimester it is unknown whether a low DHA supply early during gestation compromises embryonic brain development. Most intervention studies of prenatal DHA and infant neurodevelopment began supplementation in the second trimester, and thus our intervention at 16 weeks of gestation may have been too late if DHA is important for early structural brain development. However, the inverse association of child Beery scores with maternal erythrocytes $22: 4 n-6$ and 22 : 5n-6 may suggest that visual-motor integration development is sensitive to low prenatal DHA, consistent with the time course of brain maturation, with maturation occurring in the visual cortex before the prefrontal cortex ${ }^{(52)}$. The association between the maternal and child diet may explain the discrepancy between epidemiologic and intervention studies of prenatal DHA on child outcome, as it seems reasonable to assume that mothers with low DHA intakes during pregnancy may have children with low DHA intakes. Thus, with results of several studies also suggesting that the DHA supply in childhood is associated with brain development after birth, separating the prenatal and postnatal effects of DHA supplies on brain development is a challenge. Regardless, evidence that even short-term DHA supplementation in children improved multiple areas of neural function ${ }^{(27,29,30,33)}$ raises the possibility that the child's DHA intake and DHA status at 5.75 years may affect neurological performance.

\section{Acknowledgements}

The authors would like to acknowledge Dr Sheila Innis, whose infectious passion for research and discovery led to numerous advances in our understanding of early-life nutrition. She was a dedicated mentor and will be missed dearly by her trainees and colleagues. The authors would also like to offer the gratitude to the parents and children who dedicated their time and effort to participate in the study. Finally, the authors would like to acknowledge Roger Dyer, Janette King for their assistance with sample analysis and Kelly Richardson for conducting the developmental assessments of the children.

This research was funded by the Canadian Institutes for Health Research grant no. MOP 84248. The funders had no role in study design, data collection and analysis, decision to publish or preparation of the manuscript.

K. A. M. and S. M. I. designed the research questions and the study; K. A. M. and S. M. I. conducted the research; K. A. M., S. M. I. and R. E. analysed the data and wrote the manuscript, and S. M. I. passed away before the completion of the final draft of the manuscript. K. A. M. and R. E. read and 
approved the final manuscript and take responsibility for the published paper.

None of the authors has any conflicts of interest to declare.

\section{Supplementary material}

For supplementary material/s referred to in this article, please visit https://doi.org/10.1017/S0007114517003531

\section{References}

1. Lamptey MS \& Walker BL (1976) A possible essential role for dietary linolenic acid in the development of the young rat. J Nutr 106, 86-93.

2. Farquharson J, Jamieson EC, Abbasi KA, et al. (1995) Effect of diet on the fatty acid composition of the major phospholipids of infant cerebral cortex. Arch Dis Child 72, 198-203.

3. Neuringer M, Connor WE, Van Petten C, et al. (1984) Dietary omega- 3 fatty acid deficiency and visual loss in infant rhesus monkeys. J Clin Invest 73, 272-276.

4. Reisbick S, Neuringer M, Gohl E, et al. (1997) Visual attention in infant monkeys: effects of dietary fatty acids and age. Dev Psychol 33, 387-395.

5. Judge MP, Harel O \& Lammi-Keefe CJ (2007) A docosahexaenoic acid-functional food during pregnancy benefits infant visual acuity at four but not six months of age. Lipids $\mathbf{4 2}$, $117-122$.

6. Helland IB, Smith L, Saarem K, et al. (2003) Maternal supplementation with very-long-chain $n$-3 fatty acids during pregnancy and lactation augments children's IQ at 4 years of age. Pediatrics 111, e39-e44.

7. Dunstan JA, Simmer K, Dixon G, et al. (2008) Cognitive assessment of children at age 2(1/2) years after maternal fish oil supplementation in pregnancy: a randomised controlled trial. Arch Dis Child Fetal Neonatal Ed 93, 45-50.

8. Helland IB, Saugstad OD, Smith L, et al. (2001) Similar effects on infants of $n-3$ and $n-6$ fatty acids supplementation to pregnant and lactating women. Pediatrics 108, E82.

9. Makrides M, Gibson RA, McPhee AJ, et al. (2010) Effect of DHA supplementation during pregnancy on maternal depression and neurodevelopment of young children: a randomized controlled trial. JAMA 304, 1675-1683.

10. Smithers LG, Gibson RA \& Makrides M (2011) Maternal supplementation with docosahexaenoic acid during pregnancy does not affect early visual development in the infant: a randomized controlled trial. Am J Clin Nutr 93, 1293-1299.

11. Gould JF, Treyvaud K, Yelland LN, et al. (2017) Seven-year follow-up of children born to women in a randomized trial of prenatal DHA supplementation. JAMA 317, 1173-1175.

12. Makrides M, Gould JF, Gawlik NR, et al. (2014) Four-year follow-up of children born to women in a randomized trial of prenatal DHA supplementation. JAMA 311, 1802-1804.

13. Agostoni C, Trojan S, Bellu R, et al. (1995) Neurodevelopmental quotient of healthy term infants at 4 months and feeding practice: the role of long-chain polyunsaturated fatty acids. Pediatr Res 38, 262-266.

14. Birch EE, Garfield S, Castaneda Y, et al. (2007) Visual acuity and cognitive outcomes at 4 years of age in a double-blind, randomized trial of long-chain polyunsaturated fatty acidsupplemented infant formula. Early Hum Dev 83, 279-284.

15. Hoffman DR, Birch EE, Castaneda YS, et al. (2003) Visual function in breast-fed term infants weaned to formula with or without long-chain polyunsaturates at 4 to 6 months: a randomized clinical trial. J Pediatr 142, 669-677.
16. Colombo J, Carlson SE, Cheatham CL, et al. (2011) Long-chain polyunsaturated fatty acid supplementation in infancy reduces heart rate and positively affects distribution of attention. Pediatr Res 70, 406-410.

17. Drover JR, Hoffman DR, Castaneda YS, et al. (2011) Cognitive function in 18-month-old term infants of the DIAMOND study: a randomized, controlled clinical trial with multiple dietary levels of docosahexaenoic acid. Early Hum Dev 87, 223-230.

18. Carlson SE, Werkman SH, Rhodes PG, et al. (1993) Visual-acuity development in healthy preterm infants: effect of marine-oil supplementation. Am J Clin Nutr 58, 35-42.

19. Osterhues A, Ali NS \& Michels KB (2013) The role of folic acid fortification in neural tube defects: a review. Crit Rev Food Sci Nutr 53, 1180-1190.

20. Cao XY, Jiang XM, Dou ZH, et al. (1994) Timing of vulnerability of the brain to iodine deficiency in endemic cretinism. $N$ Engl J Med 331, 1739-1744.

21. Innis SM (2003) Perinatal biochemistry and physiology of long-chain polyunsaturated fatty acids. J Pediatr 143, S1-S8.

22. Martinez M (1992) Tissue levels of polyunsaturated fatty acids during early human development. J Pediatr 120, S129-S138.

23. Coti Bertrand P, O'Kusky JR \& Innis SM (2006) Maternal dietary (n-3) fatty acid deficiency alters neurogenesis in the embryonic rat brain. J Nutr 136, 1570-1575.

24. Chalon S (2006) Omega-3 fatty acids and monoamine neurotransmission. Prostaglandins Leukot Essent Fatty Acids 75, 259-269.

25. Dekaban AS (1978) Changes in brain weights during the span of human life: relation of brain weights to body heights and body weights. Ann Neurol 4, 345-356.

26. Levitt P (2003) Structural and functional maturation of the developing primate brain. J Pediatr 143, S35-S45.

27. Dalton A, Wolmarans P, Witthuhn RC, et al. (2009) A randomised control trial in schoolchildren showed improvement in cognitive function after consuming a bread spread, containing fish flour from a marine source. Prostaglandins Leukot Essent Fatty Acids 80, 143-149.

28. Ryan AS \& Nelson EB (2008) Assessing the effect of docosahexaenoic acid on cognitive functions in healthy, preschool children: a randomized, placebo-controlled, double-blind study. Clin Pediatr 47, 355-362.

29. Sinn N, Cooper P \& O'Dea K (2011) Fish oil supplementation, learning and behaviour in Indigenous Australian children from a remote community school: a pilot feasibility study. Aust NZJ Public Health 35, 493-494.

30. Richardson AJ, Burton JR, Sewell RP, et al. (2012) Docosahexaenoic acid for reading, cognition and behavior in children aged 7-9 years: a randomized, controlled trial (the DOLAB Study). PLOS ONE 7, e43909.

31. Kirby A, Woodward A, Jackson S, et al. (2010) A double-blind, placebo-controlled study investigating the effects of omega-3 supplementation in children aged 8-10 years from a mainstream school population. Res Dev Disabil 31, 718-730.

32. Baym CL, Khan NA, Monti JM, et al. (2014) Dietary lipids are differentially associated with hippocampal-dependent relational memory in prepubescent children. Am J Clin Nutr 99, 1026-1032.

33. McNamara RK, Able J, Jandacek R, et al. (2010) Docosahexaenoic acid supplementation increases prefrontal cortex activation during sustained attention in healthy boys: a placebocontrolled, dose-ranging, functional magnetic resonance imaging study. Am J Clin Nutr 91, 1060-1067.

34. Mulder KA, King DJ \& Innis SM (2014) Omega-3 fatty acid deficiency in infants before birth identified using a randomized trial of maternal DHA supplementation in pregnancy. PLOS ONE 9, e83764. 
35. Brown L (1997) Test of Nonverbal Intelligence, vol. 3. Austin TX: Pro Ed.

36. Liou YA, King DJ, Zibrik D, et al. (2007) Decreasing linoleic acid with constant alpha-linolenic acid in dietary fats increases $(n-3)$ eicosapentaenoic acid in plasma phospholipids in healthy men. J Nutr 137, 945-952.

37. Kaufman AS \& Kaufman NL (2004) Kaufman Assessment Battery for Children, vol. 2. San Antonio, TX: Pearson Education.

38. Dunn LM \& Dunn DM (2007) Peabody Picture Vocabulary Test, vol. 4. San Antonio, TX: Pearson Education.

39. Beery KE, Buktenica NA \& Beery NA (2004) The BeeryBuktenica Developmental Test of Visual Motor Integration (VMI), vol. 5. Minneapolis, MN: NCS Pearson Inc.

40. Leark RA GL, Kindschi CL, Dupuy TR, et al. (2007) Test of Variables of Attention. Los Alamitos, CA: The TOVA Company.

41. Brion MJ, Ness AR, Rogers I, et al. (2010) Maternal macronutrient and energy intakes in pregnancy and offspring intake at $10 \mathrm{y}$ : exploring parental comparisons and prenatal effects. Am J Clin Nutr 91, 748-756.

42. Oliveria SA, Ellison RC, Moore LL, et al. (1992) Parent-child relationships in nutrient intake: the Framingham Children's Study. Am J Clin Nutr 56, 593-598.

43. Deroma L, Valent F, Parpinel M, et al. (2013) Comparison of seafood consumption in a group of Italian mother-child pairs. $J$ Health Popul Nutr 31, 455-461.

44. Zuercher JL, Wagstaff DA \& Kranz S (2011) Associations of food group and nutrient intake, diet quality, and meal sizes between adults and children in the same household: a crosssectional analysis of U.S. households. Nutr J 10, 131.

45. Gould JF, Makrides M, Colombo J, et al. (2014) Randomized controlled trial of maternal omega-3 long-chain PUFA supplementation during pregnancy and early childhood development of attention, working memory, and inhibitory control. Am J Clin Nutr 99, 851-859.
46. Helland IB, Smith L, Blomen B, et al. (2008) Effect of supplementing pregnant and lactating mothers with $n$ - 3 verylong-chain fatty acids on children's IQ and body mass index at 7 years of age. Pediatrics 122, e472-e 479 .

47. Escolano-Margarit MV, Ramos R, Beyer J, et al. (2011) Prenatal DHA status and neurological outcome in children at age 5.5 years are positively associated. J Nutr 141, $1216-1223$

48. Makrides M, Neumann MA, Byard RW, et al. (1994) Fatty acid composition of brain, retina, and erythrocytes in breast- and formula-fed infants. Am J Clin Nutr 60, 189-194.

49. Xie L \& Innis SM (2008) Genetic variants of the FADS1 FADS2 gene cluster are associated with altered $(n-6)$ and $(n-3)$ essential fatty acids in plasma and erythrocyte phospholipids in women during pregnancy and in breast milk during lactation. J Nutr 138, 2222-2228.

50. Koletzko B, Lattka E, Zeilinger S, et al. (2011) Genetic variants of the fatty acid desaturase gene cluster predict amounts of red blood cell docosahexaenoic and other polyunsaturated fatty acids in pregnant women: findings from the Avon Longitudinal Study of Parents and Children. Am J Clin Nutr 93, 211-219.

51. Martinez M \& Mougan I (1998) Fatty acid composition of human brain phospholipids during normal development. J Neurochem 71, 2528-2533.

52. Tau GZ \& Peterson BS (2010) Normal development of brain circuits. Neuropsychopharmacology 35, 147-168.

53. Keim SA \& Branum AM (2015) Dietary intake of polyunsaturated fatty acids and fish among US children 12-60 months of age. Matern Child Nutr 11, 987-998.

54. van der Wurff IS, Bakker EC, Hornstra G, et al. (2016) Association between prenatal and current exposure to selected LCPUFAs and school performance at age 7. Prostaglandins Leukot Essent Fatty Acids 108, 22-29. 